\title{
High-Fidelity Readout of Trapped-Ion Qubits
}

\author{
A. H. Myerson, D. J. Szwer, S. C. Webster, D. T. C. Allcock, M. J. Curtis, G. Imreh, J. A. Sherman, D. N. Stacey, \\ A. M. Steane, and D. M. Lucas \\ Department of Physics, University of Oxford, Clarendon Laboratory, Parks Road, Oxford OX1 3PU, United Kingdom
} (Received 12 February 2008; published 23 May 2008)

\begin{abstract}
We demonstrate single-shot qubit readout with a fidelity sufficient for fault-tolerant quantum computation. For an optical qubit stored in ${ }^{40} \mathrm{Ca}^{+}$we achieve $99.991(1) \%$ average readout fidelity in $10^{6}$ trials, using time-resolved photon counting. An adaptive measurement technique allows $99.99 \%$ fidelity to be reached in $145 \mu$ s average detection time. For ${ }^{43} \mathrm{Ca}^{+}$, we propose and implement an optical pumping scheme to transfer a long-lived hyperfine qubit to the optical qubit, capable of a theoretical fidelity of 99.95\% in $10 \mu \mathrm{s}$. We achieve $99.87(4) \%$ transfer fidelity and $99.77(3) \%$ net readout fidelity.
\end{abstract}

PACS numbers: 03.67.- a, 37.10.Ty, 42.50.Dv

A quantum computer (QC) requires qubits which can be prepared and measured accurately, and made to interact through high-quality quantum logic gates. Successful fault-tolerant operation requires certain minimum thresholds for the fidelity of state preparation, logic gates, and state measurement. Qubit readout is vital, not only for the final output of the QC, but for the error-correction essential for its operation. There is a trade-off between the error rate permitted and the number of extra qubits required to achieve fault-tolerant quantum error correction (QEC), but typical studies require errors to be below $\sim 10^{-3}$ for realistic implementations [1,2]. A correction network typically uses more gates than individual qubit readouts, so readout error is less critical than gate error [2]. On the other hand, precise readout can be used to compensate for gate error. It is also crucial in a measurement-based QC [3].

Trapped-ion QC approaches generally use qubits based either on a hyperfine transition within the ground atomic level [4-8], or on an optical transition between ground and metastable states [9]. State preparation is implemented by optical pumping. State measurement is achieved by repeatedly exciting a cycling transition which involves one of the qubit states but not the other, and measuring whether or not the ion fluoresces [10]. The accuracy with which the measurement can be performed depends on the rate at which fluorescence photons can be detected for the "bright" qubit state, compared with the rate at which the "dark" state gets pumped to the bright manifold (e.g., by off-resonant excitation), or vice versa. The fact that many fluorescence photons can typically be emitted by the ion before the undesired pumping process occurs allows for high-fidelity single-shot measurement even though the absolute photon collection efficiency can be poor (typically $\sim 0.1 \%$ ). Measurement fidelities of $98 \%-99 \%$ have been reported $[4,6,7,9]$. In recent work, an optical qubit was measured indirectly by repetitive measurement on an ancilla qubit held in the same ion trap, yielding a fidelity of 99.94\% in $~ 12$ ms [11].

In this Letter, we report direct high-fidelity measurement of first an optical qubit stored in the $\left(4 S_{1 / 2}, 3 D_{5 / 2}\right)$ levels [12] of a single ${ }^{40} \mathrm{Ca}^{+}$ion (Fig. 1, right) and second a hyperfine qubit stored in the $S_{1 / 2}(F=3, F=4)$ sublevels of a ${ }^{43} \mathrm{Ca}^{+}$ion (Fig. 3, right), where we first map the hyperfine qubit to the ${ }^{43} \mathrm{Ca}^{+}$optical qubit. Readout is achieved by driving the $\left(S_{1 / 2} \leftrightarrow P_{1 / 2} \leftrightarrow D_{3 / 2}\right)$ manifold and detecting the fluorescence from the $P_{1 / 2} \rightarrow S_{1 / 2}$ decay: fluorescence indicates the qubit was initially in the bright $S_{1 / 2}$ state, absence of fluorescence that the qubit was in the dark metastable $D_{5 / 2}$ state (lifetime $\tau=1168(7) \mathrm{ms}$ [13]). We discuss state inference by simple photon-count thresholding and by maximum likelihood methods using time-resolved detection. The latter are applicable to a wide range of physical qubits [14].

For the ${ }^{40} \mathrm{Ca}^{+}$measurements, a single ion is held in a Paul trap [13], Doppler cooled on the $S_{1 / 2} \leftrightarrow P_{1 / 2}$ transition by a $397 \mathrm{~nm}$ laser and repumped on $D_{3 / 2} \leftrightarrow P_{1 / 2}$ by an $866 \mathrm{~nm}$ laser. A photomultiplier (PMT) detects fluorescence from the ion with a net efficiency $0.22(2) \%$. The ratio of ion fluorescence to background scattered laser light has a maximum value $\approx 690$ at low $397 \mathrm{~nm}$ intensity, but optimum readout fidelity is achieved at higher intensity because the increased fluorescence can be detected more rapidly compared with $\tau$. Mean photon-count rates for fluorescence $\left(R_{B}\right)$ and background $\left(R_{D}\right)$ in this experiment were $R_{B}=55800 \mathrm{~s}^{-1}$ and $R_{D}=442 \mathrm{~s}^{-1}$ (including the PMT dark count rate of $8.2 \mathrm{~s}^{-1}$ at $20^{\circ} \mathrm{C}$ ).

The experimental sequence consists of preparing and measuring each qubit state in turn, and comparing the measurement outcome with the known preparation. To prepare the $D_{5 / 2}$ "shelf" state a $393 \mathrm{~nm}$ beam is switched on to drive $S_{1 / 2} \leftrightarrow P_{3 / 2}$ for $1 \mathrm{~ms}$; spontaneous decay populates $D_{5 / 2}$ (the $866 \mathrm{~nm}$ beam is left on to empty $D_{3 / 2}$ ). The rate of transfer to the shelf was measured to be $[12(2) \mu \mathrm{s}]^{-1}$. The $393 \mathrm{~nm}$ beam is extinguished and, 20(2) $\mu$ s later, we start to collect PMT counts. Photons are counted for a bin time of $2 \mathrm{~ms}$, which is divided into 200 sub-bins of duration $t_{s}=10 \mu \mathrm{s}$. Two alternately gated $10 \mathrm{MHz}$ counters are used, so that there is negligible $(<50 \mathrm{~ns})$ dead time between sub-bins. The counts $n_{i}$ from each sub-bin $i$ are available to the control computer 


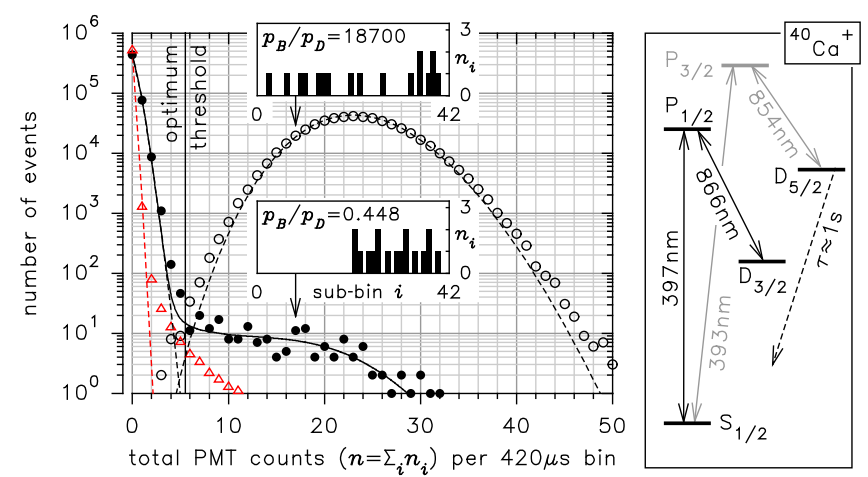

FIG. 1 (color online). ${ }^{40} \mathrm{Ca}^{+}$photon-count histograms with

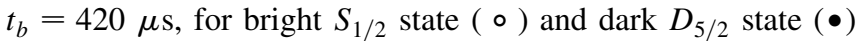
preparations. Above-threshold events in the dark histogram give $\epsilon_{D}$; below-threshold events in the bright histogram give $\epsilon_{B}$. Insets show time-resolved counts for two individual 17-photon events, one from each histogram, with likelihood ratios $p_{B} / p_{D}$. A PMT dark count histogram is also shown $(\triangle)$, normalized to the same area. Dashed curves are Poisson distributions with the same means as the histograms. The solid curve is the expected $D_{5 / 2}$ distribution taking into account spontaneous decay.

$\sim 5 \mu$ s after the end of the sub-bin, should they be required for real-time processing, and are recorded in time order. Next, the ion is prepared in $S_{1 / 2}$ by driving $D_{5 / 2} \leftrightarrow P_{3 / 2}$ for $\sim 3 \mathrm{~ms}$ with an $854 \mathrm{~nm}$ laser beam, after which fluorescence is collected for a second $2 \mathrm{~ms}$ bin.

This sequence was repeated to give $2^{20} \approx 10^{6}$ trials, in half of which the ion was prepared in the dark $D_{5 / 2}$ state and in half of which it was prepared in the bright $S_{1 / 2}$ state. We define the average readout error to be $\epsilon=\frac{1}{2}\left(\epsilon_{B}+\epsilon_{D}\right)$, where $\epsilon_{B}$ is the fraction of experiments in which an ion prepared in the bright state was detected to be dark, and similarly for $\epsilon_{D}$. In the case of the dark state experiments, there are two small preparation errors arising from (i) the finite shelving rate of the $393 \mathrm{~nm}$ laser and (ii) the probability of spontaneous decay during the $20 \mu$ s delay between state preparation and the start of the detection period. These give a contribution to $\epsilon_{D}$ of $(12 \mu \mathrm{s}+$ $20 \mu \mathrm{s}) / \tau=0.28(2) \times 10^{-4}$. Known state preparation errors for the bright state are negligible, so the net contribution to $\epsilon$ is $0.14(1) \times 10^{-4}$. Values of $\epsilon$ given below are after subtraction of this quantity.

Histograms of the number of photons $n=\sum_{i=1}^{N} n_{i}$ collected for the bright and dark state preparations are shown in Fig. 1. Here the first $N=42$ sub-bins of the detection period were summed for a total detection time of $t_{b}=$ $N t_{s}=420 \mu \mathrm{s}$; this choice of $t_{b}$ and a threshold at $n_{c}=5 \frac{1}{2}$ counts optimize the discrimination between the bright and dark histograms. The error $\epsilon$ as a function of $t_{b}$ is shown in Fig. 2, for $N=1 \ldots 100$. As $t_{b}$ increases, $\epsilon$ first drops rapidly due to decreasing overlap between the two histograms, to a minimum $\epsilon=1.8(1) \times 10^{-4}$ at $t_{b}=420 \mu \mathrm{s}$, then rises slowly because of the increasing probability of decay from $D_{5 / 2}$ during $t_{b}$.
At the optimum $t_{b}$, the majority of the error is due to the above-threshold events in the dark histogram: $\epsilon_{D}=$ $3.2(3) \times 10^{-4}$. These mostly arise due to spontaneous decay during $t_{b}$, but we also observe above-threshold events from non-Poissonian PMT dark counts. A PMT dark count distribution is also shown in Fig. 1: the highly non-Poissonian tail in the histogram probably arises as a result of cosmic rays [15]. The slow time scale of luminescence in the PMT envelope excited by the cosmic rays makes it difficult to eliminate these events entirely. We estimate that, for the threshold method, cosmic ray events account for $\sim 20 \%$ of $\epsilon_{D}$.

The threshold method does not make use of the arrivaltime information of the photons. Using this information, we can hope to identify some of the events where decay from $D_{5 / 2}$ occurs during $t_{b}$ : we may detect $>n_{c}$ total counts, but if these are clustered near the end of the bin this suggests a decay event rather than a bright ion (compare Fig. 1 insets). The use of time-resolved measurement in the context of qubit readout was suggested in [4] and modeled theoretically in $[14,16]$; we follow a similar maximum likelihood treatment to Langer [16].

We calculate the likelihood $p_{B}$ that a given set of subbins $\left\{n_{i}\right\}$ could have been generated by a bright ion, and compare this with the likelihood $p_{D}$ that $\left\{n_{i}\right\}$ arose from an ion that was dark at the start of the detection period. If $p_{B}>p_{D}$ we infer that the ion was bright and vice versa. $p_{B}=P\left(\left\{n_{i}\right\} \mid\right.$ bright $)$ is given simply by the product of probabilities $p_{B}=\prod_{i=1}^{N} B\left(n_{i}\right)$ where $B\left(n_{i}\right)$ is the probability of observing $n_{i}$ counts from a bright ion [in the ideal case $B\left(n_{i}\right)$ is a Poisson distribution with mean count per sub-bin $\left.R_{B} t_{s}\right]$. The calculation of $p_{D}=P\left(\left\{n_{i}\right\} \mid\right.$ dark $)$ is more involved, because we must sum over the possibilities that the ion decayed after or during the detection time $t_{b}=$ $N t_{s}$. To a good approximation

$$
p_{D}=\left(1-\frac{t_{b}}{\tau}\right) \prod_{i=1}^{N} D\left(n_{i}\right)+\left(\frac{t_{s}}{\tau}\right) \sum_{j=1}^{N} \prod_{i=1}^{j-1} D\left(n_{i}\right) \prod_{i=j}^{N} B\left(n_{i}\right)
$$

where $\left(1-t_{b} / \tau\right)$ is the probability the ion decays after the last sub-bin, $\left(t_{s} / \tau\right)$ the probability it decays during any particular sub-bin $j$, and $D\left(n_{i}\right)$ the background count distribution with mean $R_{D} t_{s}$. Using a recursion relation

$$
\begin{gathered}
p_{D}=\left(1-t_{b} / \tau\right) M_{N}+\left(t_{s} / \tau\right) S_{N} ; \\
M_{0}=1, \quad M_{k}=M_{k-1} D\left(n_{k}\right) ; \\
S_{0}=0, \quad S_{k}=\left(S_{k-1}+M_{k-1}\right) B\left(n_{k}\right)
\end{gathered}
$$

reduces the calculation of $p_{D}$ from $O\left(N^{2}\right)$ to $O(N)$ operations, making real-time readout much faster. We have made the simplifying approximations that $t_{s}, t_{b} \ll \tau$, and that the mean count rate changes from $R_{D}$ to $R_{B}$ at the start of the sub-bin $j$ in which the ion decays; similar expressions are found if these approximations are not made, with negligible effect on the results. 


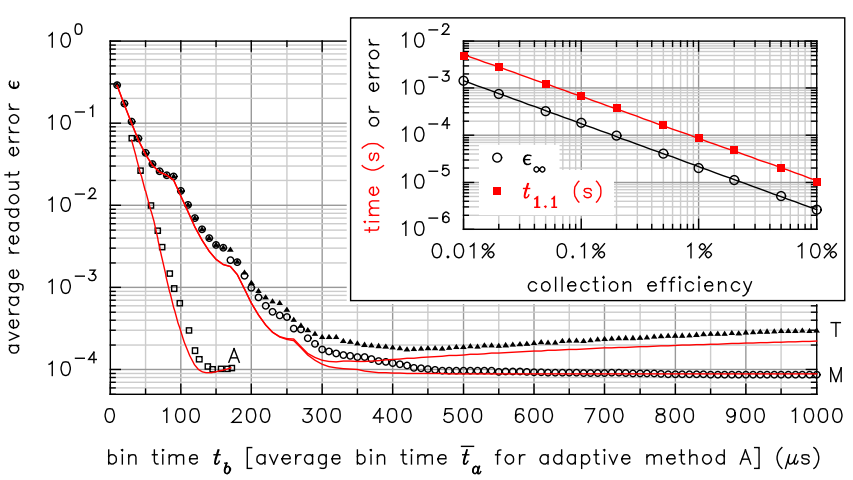

FIG. 2 (color online). Average readout error $\epsilon$ versus readout time for the ${ }^{40} \mathrm{Ca}^{+}$optical qubit, for three different analysis methods. Analyses of the experimental data are plotted as symbols; statistical uncertainty is at most the size of the symbols. The accompanying curves are simulations of $10^{9}$ trials using ideal Poisson statistics. Cusps arise from the discrete nature of photon counting. T: photon-count threshold method, where the threshold $n_{c}$ was optimized for each bin time. M: maximum likelihood method. A: adaptive maximum likelihood method. Inset: Simulations using method $\mathbf{M}$, showing the asymptotic average error $\epsilon_{\infty}$ and the time $t_{1.1}$ required to reach $\epsilon=1.1 \epsilon_{\infty}$ as functions of the net photon collection efficiency.

The results of applying the maximum likelihood method to the same data set are also shown in Fig. 2. For $B\left(n_{i}\right)$ and $D\left(n_{i}\right)$, Poisson distributions were convolved with the PMT dark count distribution [17]. We see that for $t_{b} \gtrsim 200 \mu \mathrm{s}$ the maximum likelihood method gives a lower error than the threshold method, tending to an asymptotic value $\epsilon=$ $0.87(11) \times 10^{-4}$ [with $\epsilon_{D}=1.5(2) \times 10^{-4}$ ], or a fidelity $99.9913(11) \%$. Furthermore, the maximum likelihood method does not require a precise choice of parameters $\left(n_{c}, t_{b}\right)$ which must be determined from control data; we only need to know the independently measured rates $R_{B}$ and $R_{D}$, and to choose a sufficiently long $t_{b}$. The observed error is compared with that from a simulation of $10^{9}$ trials using ideal Poisson statistics in Fig. 2: although at short $t_{b}$ the experimental $\epsilon$ is greater due to super-Poissonian noise in the data, at long $t_{b}$ it converges to the simulation's asymptote at $\epsilon=0.89 \times 10^{-4}$. We conclude that, at optimum $t_{b}$, the maximum likelihood method is less sensitive to experimental noise (e.g., cosmic ray events, drift in $R_{B}$ ) than the threshold method.

We now discuss faster detection by using an adaptive version of the maximum likelihood technique [11]. In the preceding discussion, the detection bin length $t_{b}=N t_{s}$ was fixed and we evaluated the likelihoods $p_{B}, p_{D}$ at the end of the bin, deciding that the ion was dark if $p_{D}>p_{B}$. However, the absolute values of $p_{B}$ and $p_{D}$ also contain useful information. The estimated error probability $e_{D}$ that we have incorrectly deduced the ion to be dark when $p_{D}>$ $p_{B}$ is given by Bayes' theorem [18]:

$$
e_{D}=1-P\left(\operatorname{dark} \mid\left\{n_{i}\right\}\right)=1-\frac{P\left(\left\{n_{i}\right\} \mid \text { dark }\right)}{P\left(\left\{n_{i}\right\}\right)}=\frac{p_{B}}{p_{B}+p_{D}}
$$

and similarly for $e_{B}$ in the case where $p_{B} \geq p_{D}$. By evaluating $p_{D}$ and $p_{B}$ at the end of each sub-bin, we can terminate the detection bin at $t_{a}<t_{b}$ when the estimated error probability $e_{B}$ or $e_{D}$ falls below some chosen cutoff threshold $e_{c}$. We also impose a cutoff time $t_{c} \leq t_{b}$ in case the error threshold is not reached; $t_{c}$ is thus the worst case readout time. The result is that, for a given desired error level, the average detection bin length $\bar{t}_{a}$ is shorter than when the bin length is fixed. If $e_{c}$ is not reached, $e_{B}$ or $e_{D}$ still quantifies confidence in the measurement outcome, useful for QEC. Using the recursion relations (1) we can evaluate $p_{B}, p_{D}$ in $<1 \mu \mathrm{s}$, while each sub-bin is in progress, so there is negligible time overhead.

For our conditions we find that, at little cost to $\epsilon$, faster detection can be obtained by omitting the effect of spontaneous decay in the analysis, i.e., using $p_{D}=\prod_{i=1}^{N} D\left(n_{i}\right)$. An adaptive analysis of the same data set is shown in Fig. 2, for $t_{c}=500 \mu \mathrm{s}$ and a range of threshold values $10^{-6} \leq$ $e_{c} \leq 10^{-1}$. For each $e_{c}$ we plot the experimentally measured average error $\epsilon$ against $\bar{t}_{a}$. For an average readout time of $\bar{t}_{a}=145 \mu \mathrm{s}$, the error reaches $\epsilon=1.0(1) \times 10^{-4}$, which is about 35 times lower than the nonadaptive maximum likelihood method, or over 3 times as fast for the same $\epsilon$. The average readout time for the bright (dark) state is $72 \mu \mathrm{s}(219 \mu \mathrm{s})$.

For all of the analysis methods described, the bright $S_{1 / 2}$ state can be detected more accurately than the dark $D_{5 / 2}$ state because the known bright $\rightarrow$ dark transfer rate is so low (we measure it to be $<10^{-3} \mathrm{~s}^{-1}$ ). The data imply the bright state can be detected with over $99.9998 \%$ fidelity, $\epsilon_{B}<2 \times 10^{-6}$, in average time $\bar{t}_{a}=91 \mu$ s [while retaining $\epsilon_{D}=2.5(2) \times 10^{-4}$ in $\left.\bar{t}_{a}=292 \mu \mathrm{s}\right]$. This asymmetric readout error could be exploited for QEC.

The optical qubit offers high-fidelity readout but it suffers from two drawbacks: a finite lifetime $\tau$ and the need for high frequency stability in the optical domain. Qubits stored in hyperfine ground states avoid these problems and exhibit some of the longest coherence times ever measured $[5,8]$. We turn now to the implementation of state detection for a qubit stored in the $|\uparrow\rangle=S_{1 / 2}^{3,+3}$ and $|\downarrow\rangle=S_{1 / 2}^{4,+4}$ hyperfine ground states of ${ }^{43} \mathrm{Ca}^{+}$(where the superscripts give the quantum numbers $F, M_{F}$ ). For readout, we first map this hyperfine qubit to the optical qubit by state-selective transfer $|\downarrow\rangle \rightarrow D_{5 / 2}$.

We Doppler cool a single ${ }^{43} \mathrm{Ca}^{+}$ion on the $S_{1 / 2}^{3} \leftrightarrow P_{1 / 2}^{4}$ and $S_{1 / 2}^{4} \leftrightarrow P_{1 / 2}^{4}$ transitions. State preparation of $|\downarrow\rangle$ is by optical pumping with $\sigma^{+}$polarized light on the same transitions. Preparation of $S_{1 / 2}^{3, M_{F}}$ is achieved by extinguishing the $S_{1 / 2}^{3} \leftrightarrow P_{1 / 2}^{4}$ beam before the $S_{1 / 2}^{4} \leftrightarrow P_{1 / 2}^{4}$ beam: this prepares a statistical mixture of $M_{F}$ states rather than $|\uparrow\rangle$ but the calculated effect on the measured readout error is $\left\langle 10^{-4}\right.$. To map $\left.\mid \downarrow\right\rangle$ to the $D_{5 / 2}$ shelf the ion is illuminated with $393 \mathrm{~nm} \sigma^{+}$light which drives $|\downarrow\rangle \leftrightarrow P_{3 / 2}^{5,+5}$. This results in optical pumping to $D_{5 / 2}$ or $D_{3 / 2}$ with branching ratios $5.3 \%$ and $0.63 \%$, respectively [13]. Light 


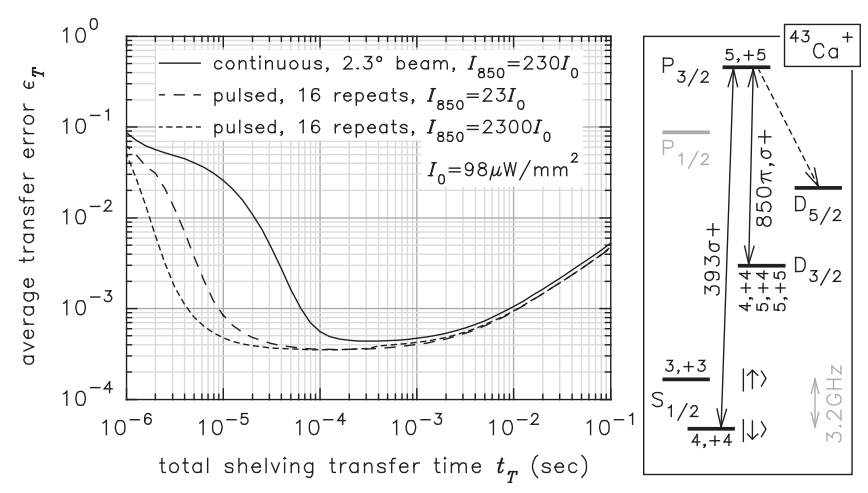

FIG. 3. Rate equation simulations for the ${ }^{43} \mathrm{Ca}^{+}$shelving transfer $(|\uparrow\rangle,|\downarrow\rangle) \rightarrow\left(|\uparrow\rangle, D_{5 / 2}\right)$. The optimum average shelving transfer error $\epsilon_{T}$ is shown versus total time $t_{T}$ allowed for the transfer, for both continuous and pulsed methods. The $393 \mathrm{~nm}$ intensity and (in the pulsed method) all pulse durations were numerically optimized for each $t_{T}$. The error increases at short $t_{T}$ due to higher $393 \mathrm{~nm}$ intensity causing off-resonant shelving of $|\uparrow\rangle$; at long $t_{T}$ the error increases because of decay from the $D_{5 / 2}$ shelf. The minimum error is $3.5 \times 10^{-4}$ at $t_{T}=160 \mu \mathrm{s}$; for $t_{T}=$ $10 \mu \mathrm{s}$ an error of $4.8 \times 10^{-4}$ is available. Right: Simplified ${ }^{43} \mathrm{Ca}^{+}$level diagram; states relevant to the shelving transfer are labeled by $F, M_{F}$.

at $850 \mathrm{~nm}$ containing $\sigma^{+}$and $\pi$ polarizations is used to empty the relevant $D_{3 / 2}$ states via the $P_{3 / 2}^{5,+5}$ state (Fig. 3, right). Assuming perfect repumping from $D_{3 / 2}$, the situation reduces to that for ions without low-lying $D$ levels, but with the important difference that we only need to drive enough transitions to transfer $|\downarrow\rangle$ to the shelf (rather than to collect fluorescence) before off-resonant excitation of $|\uparrow\rangle$ occurs; since the $P_{3 / 2} \rightarrow D_{5 / 2}$ branching ratio is much greater than typical photon collection efficiencies this gives a significant advantage.

To optimize parameters the shelving transfer process was modeled by rate equations applied to the entire 144state $(4 S, 4 P, 3 D)$ manifold. The optimum shelving method would be a repeated sequence of three laser pulses (393 nm $\sigma^{+}, 850 \mathrm{~nm} \sigma^{+}, 850 \mathrm{~nm} \pi$ ), since then there is negligible probability of the ion decaying to $|\uparrow\rangle$. The main limitation is off-resonant (by $3.1 \mathrm{GHz}$ ) excitation of $|\uparrow\rangle \leftrightarrow$ $P_{3 / 2}^{4,+4}$. Continuous excitation allows similar fidelity if the $850 \mathrm{~nm} \pi$ component is weak (though with slower shelving; see Fig. 3). Accordingly, in the experiment, we used a single circularly polarized $850 \mathrm{~nm}$ beam travelling at a small angle $\left(2.3^{\circ}\right)$ to the quantization axis, giving polarization components with intensities $\left(I_{\sigma^{+}}, I_{\pi}, I_{\sigma^{-}}\right)=$ $\left(0.9992,0.0008,2 \times 10^{-7}\right) \times 230(70) I_{0}$, where the saturation intensity $I_{0}=98 \mu \mathrm{W} / \mathrm{mm}^{2}$. The shelving transfer was accomplished with a single simultaneous $393 \mathrm{~nm}+$ $850 \mathrm{~nm}$ pulse, with duration set to the optimum value predicted by the model, $t_{T}=400 \mu \mathrm{s}$. No improvement was found by varying $t_{T}$ or by using alternating 393 and $850 \mathrm{~nm}$ pulses (to avoid two-photon effects, which are not modeled by the rate equations). After shelving, state de- tection proceeds as for ${ }^{40} \mathrm{Ca}^{+}$; photon-count thresholding with $t_{b}=2 \mathrm{~ms}$ was used as the optical qubit readout is not the limiting factor.

The net average readout error for the hyperfine qubit was measured from 20000 trials to be $\epsilon_{\mathrm{hfs}}=\frac{1}{2}\left(\epsilon_{\uparrow}+\epsilon_{\downarrow}\right)=$ $2.3(3) \times 10^{-3}$, a fidelity of $99.77(3) \%$. [The error for the $|\uparrow\rangle$ state was $\epsilon_{\uparrow}=1.7(4) \times 10^{-3}$.] A separate experiment measured the optical qubit readout error to be $1.0(2) \times$ $10^{-3}$, significantly poorer than for ${ }^{40} \mathrm{Ca}^{+}$due to a lower fluorescence rate $R_{B}=7500 \mathrm{~s}^{-1}$ (caused partly by coherent population trapping effects, which could be eliminated by polarization modulation techniques [19]). This implies an average error $\epsilon_{T}=1.3(4) \times 10^{-3}$ for the shelving transfer. This is somewhat above the modeled value of $0.44 \times 10^{-3}$; possible reasons for this include imperfect circular polarization of the $850 \mathrm{~nm}$ beam, imperfect population preparation, and broadening of the $393 \mathrm{~nm}$ transition (e.g., due to finite laser linewidth).

In conclusion, we demonstrate fast, direct, single-shot readout from optical and hyperfine trapped-ion qubits at fidelities comparable with those required for a faulttolerant QC. An adaptive detection method reduced the optical qubit readout error by a factor $\approx 35$ for a given average detection time, reaching $99.99 \%$ fidelity in $145 \mu \mathrm{s}$, with negligible time overhead due to the classical control system. A simple, robust method for readout of hyperfine qubits, capable of comparable fidelity and speed, was proposed and implemented. An increase in the photon collection efficiency by an order of magnitude, to $2 \%$, would speed up both readout methods, and reduce the optical qubit readout error, by a similar factor (Fig. 2, inset).

We thank C. Langer and J. P. Home for helpful discussions. This work was supported by EPSRC (QIP IRC), DTO (Ref. 47968-PH-QC), the European Commission (SCALA, MicroTrap), and the Royal Society.

[1] E. Knill, Nature (London) 434, 39 (2005).

[2] A. M. Steane, Quantum Inf. Comput. 7, 171 (2007).

[3] M. A. Nielsen, Rep. Math. Phys. 57, 147 (2006).

[4] C. Monroe et al., in Atomic Physics 17, edited by P. De Natale (AIP Press, New York, 2001), p. 173.

[5] C. Langer et al., Phys. Rev. Lett. 95, 060502 (2005).

[6] M. Acton et al., Quantum Inf. Comput. 6, 465 (2006).

[7] S. Olmschenk et al., Phys. Rev. A 76, 052314 (2007).

[8] D. M. Lucas et al., arXiv:quant-ph/0710.4421v1.

[9] C. Wunderlich et al., J. Mod. Opt. 54, 1541 (2007).

[10] D. J. Wineland et al., Opt. Lett. 5, 245 (1980).

[11] D. B. Hume et al., Phys. Rev. Lett. 99, 120502 (2007).

[12] The readout method is not sensitive to the choice of $M_{J}$.

[13] P. Barton et al., Phys. Rev. A 62, 032503 (2000).

[14] J. Gambetta et al., Phys. Rev. A 76, 012325 (2007).

[15] M. C. Teich et al., Phys. Rev. D 36, 2649 (1987).

[16] C. Langer, Ph.D. thesis, University of Colorado, 2006.

[17] We neglect time correlations caused by the cosmic rays.

[18] T. Bayes, Phil. Trans. R. Soc. London 53, 370 (1763).

[19] M. G. Boshier et al., Appl. Phys. B 71, 51 (2000). 